\title{
Stannane-Free Chemoselective
}

\section{Hydrodehalogenation of 4-Halo-}

\section{Tetrahydropyrans: Scope and Application to Natural Product Synthesis}

\author{
Kok-Ping Chan, Yvonne Hui Ling, Jocelyn Li-Ting Chan, \\ and Teck-Peng Loh * \\ Division of Chemistry and Biological Chemistry \\ School of Physical and Mathematical Sciences \\ Blk 5, Level 3, Unit 43, 1 Nanyang Walk, Nanyang Technological University \\ Singapore 637616
}

Supplementary Material 
Stannane-Free Chemoselective Hydrodehalogenation of 4-HaloTetrahydropyrans: Scope and Application to Natural Product Synthesis

Kok-Ping Chan, Yvonne Hui Ling, Jocelyn Li-Ting Chan and Teck-Peng Loh*

Division of Chemistry and Biological Chemistry

School of Physical and Mathematical Sciences

Blk 5, Level 3, Unit 43, 1 Nanyang Walk, Nanyang Technological University

Singapore 637616

\section{Supporting Information}

General Methods

General Procedure for the Synthesis of Dehalo-tetrahydropyran

Characterization of Compounds derived from Stannane-Free Hydrodehalogenation reaction

Procedure and Characterization of Intermediates of (+)-SCH 351448 


\section{Supporting Information}

\section{General Methods}

Experiments involving moisture and/or air sensitive components were performed in oven-dried glassware. Commercial solvents and reagents were used without further purification with the following exceptions: Hexane, dichloromethane, ethyl acetate and diethylether were fractionally distilled. Azeotropic drying of starting materials or reagents was performed by the addition of the stated amount of anhydrous tetrahydrofuran, ensued by azeotropic removal of tetrahydrofuran with traces of moisture in vacuo followed by purging with nitrogen. Methanol was passed through Linde type $4 \AA$ molecular sieve before distillation.

Analytical thin layer chromatography (TLC) was performed using Merck 60 F254 precoated silica gel plate $(0.2 \mathrm{~mm}$ thickness). Subsequent to elution, plates were visualized using UV radiation $(254 \mathrm{~nm}$ ) on Spectroline Model ENF24061/F $254 \mathrm{~nm}$. Further visualization was possible by staining with basic solution of potassium permanganate or acidic solution of ceric molybdate, followed by heating on a hot plate.

Flash chromatography was performed using Merck silica gel 60 with freshly distilled solvents. Columns were typically packed as slurry and equilibrated with the appropriate solvent system prior to use.

Infrared spectra were recorded on a Shimadzu IR Prestige-21 FT-IR Spectrometer. Liquid samples were examined as film between $\mathrm{NaCl}$ salt plates.

Proton nuclear magnetic resonance spectra ( ${ }^{1} \mathrm{H}$ NMR) were recorded on a Bruker Avance 400 and 500 spectrophotometers ( $\mathrm{CDCl}_{3}$ as solvent). Chemical shifts for ${ }^{1} \mathrm{H}$ NMR spectra are reported as $\delta$ in units of parts per million (ppm) downfield from $\mathrm{SiMe}_{4}(\delta 0.0 \mathrm{ppm})$ and relative to the signal of chloroform- $d(\delta$ 
7.2600, singlet). Multiplicities were given as: s (singlet); d doublet); t (triplet); $q$ (quartet); dd (doublets of doublet); ddd (doublets of doublets of doublet); dt (doublets of triplet); td (triplets of doublet); tdd (triplets of doublets of doublets); $\mathrm{tt}$ (triplets of triplets) or $\mathrm{m}$ (multiplets). The number of protons (n) for a given resonance is indicated by $\mathrm{nH}$. Coupling constants are reported as a J value in $\mathrm{Hz}$. Carbon nuclear magnetic resonance spectra $\left({ }^{13} \mathrm{C}\right.$ NMR) are reported as $\delta$ in units of parts per million (ppm) downfield from $\mathrm{SiMe}_{4}(\delta 0.0 \mathrm{ppm})$ and relative to the signal of chloroform- $d(\delta 77.03 \mathrm{ppm}$, triplet). The proportion of diastereomers and geometric isomers was determined from the integration of ${ }^{1} \mathrm{H} N M R$ and ${ }^{13} \mathrm{C}$ NMR spectra.

Mass spectral analyses (HRMS) were carried out on a Thermo Finnigan MAT 95 XP mass spectrophotometer. Mass spectral data were reported in units of mass to charge $(\mathrm{m} / \mathrm{z})$.

Optical rotation was measured using a JASCO P-1030 Polarimeter equipped with a sodium vapor lamp at $589 \mathrm{~nm}$. Concentration is denoted as $c$ and was calculated as grams per milliliters $(\mathrm{g} / 100 \mathrm{~mL})$ whereas the solvent was indicated in parentheses ( $c$, solvent). 


\section{Experimental}

\section{General Method for the Synthesis of de-halo-tetrahydropyran}

To a $25 \mathrm{ml}$ round-bottom flask equipped with a magnetic stirring bar was charged $10 \%$ Pd in carbon (0.122 g, $0.11 \mathrm{mmol}, 0.4$ equiv.) in methanol $(2.57 \mathrm{~mL})$. 4-Halo-6cyclohexyl-tetrahydro- $2 H$-pyran $(0.28 \mathrm{mmol})$ in ethyl acetate $(0.28 \mathrm{ml})$ was added to the mixture and was allowed to stir under hydrogen atmosphere for $8 \mathrm{~h}$. Sodium bicarbonate (47 $\mathrm{mg}, 0.56 \mathrm{mmol}$ ) was the added to the reaction mixture and was stirred under hydrogen atmosphere for another 16 hours. The mixture was filtered through celite and was then concentrated in vacuo. The residue was diluted with ethyl acetate $(5 \mathrm{~mL})$ prior to washing with water $(2 \times 5 \mathrm{~mL})$ followed by brine solution $(2 \times 5 \mathrm{~mL})$. The organic layer was dried with anhydrous $\mathrm{MgSO}_{4}$ and concentrated in vacuo. The residual crude product was purified via flash column chromatography to afford the dehalotetrahydropyran.

\section{2-(4-Chloro-6-cyclohexyl-tetrahydro-2H-pyran-2-yl)ethanol (1a)}

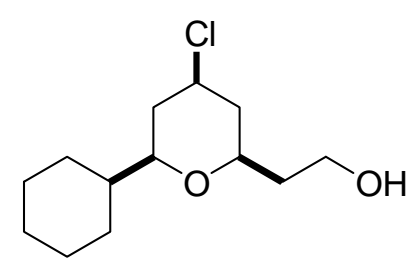

Colourless oil (54 mg, 79\% yield).

${ }^{1} \mathrm{H}$ NMR (400 MHz, $\mathrm{CDCl}_{3}$ ): $\delta 4.00(\mathrm{tt}, J=11.8,4.5 \mathrm{~Hz}, 1 \mathrm{H}), 3.80-3.75(\mathrm{~m}, 2 \mathrm{H}), 3.58-$ $3.51(\mathrm{~m}, 1 \mathrm{H}), 3.09$ (ddd, $J=11.3,6.3,1.7 \mathrm{~Hz}, 1 \mathrm{H}), 2.68(\mathrm{br} \mathrm{s}, 1 \mathrm{H}), 2.14(\mathrm{tdd}, J=12.7$, 4.2, $2.0 \mathrm{~Hz}, 1 \mathrm{H}), 2.08$ (tdd, $J=12.7,4.6,2.4 \mathrm{~Hz}, 1 \mathrm{H}), 1.86-1.77$ (m, 1H), 1.76-1.67 (m, $5 \mathrm{H}), 1.67-1.61(\mathrm{~m}, 2 \mathrm{H}), 1.45-1.34(\mathrm{~m}, 1 \mathrm{H}), 1.27-1.14(\mathrm{~m}, 4 \mathrm{H}), 1.05-0.92(\mathrm{~m}, 2 \mathrm{H})$.

${ }^{13} \mathrm{C}$ NMR $\left(100 \mathrm{MHz}, \mathrm{CDCl}_{3}\right): \delta \underline{8} 81.4,76.9,61.1,56.0,42.5,42.5,39.3,37.5,28.8,28.8$, 26.4, 26.0, 25.9.

FTIR (neat) $v_{\max }: 3373,2924,2851,1449,1080,1051,760,606 \mathrm{~cm}^{-1}$.

HRMS (EI) $m / z$ Calcd for $\mathrm{C}_{13} \mathrm{H}_{23} \mathrm{ClO}_{2}\left[\mathrm{M}^{+}\right]=246.1381$. Found: 246.1338 


\section{2-(6-Cyclohexyl-tetrahydro-2H-pyran-2-yl)ethanol (1b)}

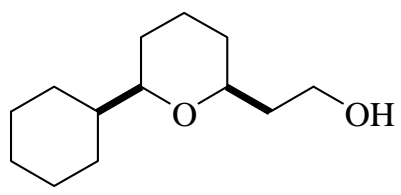

Colourless oil (55 mg, 92\% yield).

${ }^{1} \mathrm{H}$ NMR (400 MHz, $\left.\mathrm{CDCl}_{3}\right): \delta 3.78(\mathrm{t}, J=4.8 \mathrm{~Hz}, 2 \mathrm{H}), 3.58-3.50(\mathrm{~m}, 1 \mathrm{H}), 3.20$ (br s, 1H), 3.07 (ddd, $J=11.2,6.4,1.9 \mathrm{~Hz}, 1 \mathrm{H}), 1.88-1.78(\mathrm{~m}, 3 \mathrm{H}), 1.76-1.66(\mathrm{~m}, 5 \mathrm{H}), 1.65-$ $1.55(\mathrm{~m}, 4 \mathrm{H}), 1.52-1.46(\mathrm{~m}, 3 \mathrm{H}), 1.31-1.23(\mathrm{~m}, 2 \mathrm{H}), 0.97-0.88(\mathrm{~m}, 2 \mathrm{H})$.

${ }^{13} \mathrm{C} \mathrm{NMR}\left(100 \mathrm{MHz}, \mathrm{CDCl}_{3}\right): \delta 82.6,79.2,62.0,43.0,37.8,31.7,29.0,29.0,28.1,26.5$, $26.2,26.1,23.6$.

FTIR (neat) $v_{\max }: 3370,2928,2853,1449,1084,1042,733 \mathrm{~cm}^{-1}$.

HRMS (EI) $m / z$ Calcd for $\mathrm{C}_{13} \mathrm{H}_{24} \mathrm{O}_{2}\left[\mathrm{M}^{+}\right]=212.1771$. Found: 212.1763

\section{2-(2-(Benzyloxy)ethyl)-6-cyclohexyl-tetrahydro-2H-pyran (1c)}

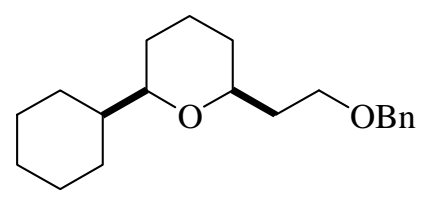

Colourless oil (63 mg, 74\% yield).

${ }^{1} \mathrm{H}$ NMR (400 MHz, $\left.\mathrm{CDCl}_{3}\right): \delta 7.37-7.31(\mathrm{~m}, 4 \mathrm{H}), 7.31-7.27(\mathrm{~m}, 1 \mathrm{H}), 4.53$ and 4.49 $(\mathrm{ABq}, J=11.8 \mathrm{~Hz}, 2 \mathrm{H}), 3.68-3.54(\mathrm{~m}, 2 \mathrm{H}), 3.46-3.37(\mathrm{~m}, 1 \mathrm{H}), 2.94$ (ddd, $J=10.7,7.6$, $1.8 \mathrm{~Hz}, 1 \mathrm{H}), 1.98-1.91(\mathrm{~m}, 1 \mathrm{H}), 1.87-1.78(\mathrm{~m}, 1 \mathrm{H}), 1.78-1.71(\mathrm{~m}, 3 \mathrm{H}), 1.70-1.67(\mathrm{~m}, 1 \mathrm{H})$, $1.66-1.56(\mathrm{~m}, 3 \mathrm{H}), 1.31-1.25$ (m, 2H), 1.24-1.15 (m, 4H), 1.15-1.13 (m, 2H), 1.01-0.82 $(\mathrm{m}, 2 \mathrm{H})$.

${ }^{13} \mathrm{C}$ NMR (100 MHz, $\left.\mathrm{CDCl}_{3}\right): \delta 138.7,128.3,127.6,127.4,82.2,74.6,73.0,67.0,43.2$, $36.8,32.0,29.3,28.9,28.6,26.7,26.3,26.1,23.9$.

FTIR (neat) $v_{\max }: 3435,3107,3086,3063,3028,2826,2853,1494,1450,1089,1043$, $733,696 \mathrm{~cm}^{-1}$.

HRMS (EI) $m / z$ Calcd for $\mathrm{C}_{20} \mathrm{H}_{30} \mathrm{O}_{2}\left[\mathrm{M}^{+}\right]=302.2240$. Found: 302.2243 . 


\section{(4-Chloro-6-cyclohexyl-tetrahydro-2H-pyran-2-yl)methanol (2a)}

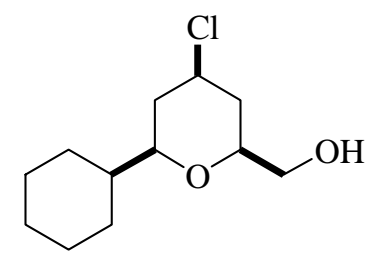

Colourless oil (42 mg, 65\% yield).

${ }^{1} \mathrm{H}$ NMR (400 MHz, $\mathrm{CDCl}_{3}$ ): $\delta 4.03(\mathrm{tt}, J=11.9,4.7 \mathrm{~Hz}, 1 \mathrm{H}), 3.65-3.58(\mathrm{~m}, 1 \mathrm{H}), 3.59$ $5.51(\mathrm{~m}, 1 \mathrm{H}), 3.48-3.41(\mathrm{~m}, 1 \mathrm{H}), 3.10(\mathrm{ddd}, J=11.2,6.4,1.6 \mathrm{~Hz}, 1 \mathrm{H}), 2.15$ (tdd, $J=$ 12.6, 4.5, $2.1 \mathrm{~Hz}, 1 \mathrm{H}), 2.08-1.99$ (m, 2H), 1.93-1.83 (m, 1H), 1.78-1.64 (m, 4H), 1.57$1.51(\mathrm{~m}, 1 \mathrm{H})$ 1.47-1.40 (m, 1H), 1.30-1.13 (m, 4H), 1.08-0.93 (m, 2H).

${ }^{13} \mathrm{C}$ NMR $\left(100 \mathrm{MHz}, \mathrm{CDCl}_{3}\right): \delta 81.0,76.9,65.7,56.1,42.7,39.4,38.3,28.9,28.6,26.5$, 26.1, 26.0.

FTIR (neat) $v_{\max }: 3412,2926,2853,1450,1087,1065,1043,764,738,610 \mathrm{~cm}^{-1}$.

HRMS (EI) $m / z$ Calcd for $\mathrm{C}_{12} \mathrm{H}_{21} \mathrm{ClO}_{2}\left[\mathrm{M}^{+}\right]=232.1225$. Found: 232.1229 .

\section{2-(Benzyloxymethyl)-6-cyclohexyl-tetrahydro-2H-pyran (2c)}

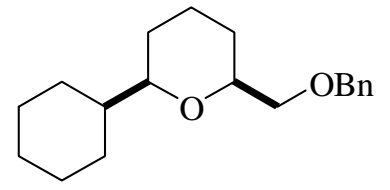

Colourless oil (38 mg, 47\% yield).

${ }^{1} \mathrm{H}$ NMR (400 MHz, $\left.\mathrm{CDCl}_{3}\right): \delta$ 7.38-7.31 (m, 4H), 7.30-7.24 (m, 1H), 4.61 and 4.56 $(\mathrm{ABq}, J=12.1 \mathrm{~Hz}, 2 \mathrm{H}), 3.54-3.48$ (m, 2H), 3.44-3.39 (m, 1H), 3.09-2.99 (m, 1H), 2.01$1.92(\mathrm{~m}, 1 \mathrm{H}), 1.91-1.82(\mathrm{~m}, 1 \mathrm{H}), 1.79-1.72(\mathrm{~m}, 1 \mathrm{H}), 1.72-1.68(\mathrm{~m}, 2 \mathrm{H}), 1.68-1.65$ (m, $1 \mathrm{H}), 1.46-1.40(\mathrm{~m}, 1 \mathrm{H}), 1.39-1.32(\mathrm{~m}, 1 \mathrm{H}), 1.30-1.18(\mathrm{~m}, 5 \mathrm{H}), 1.18-1.10(\mathrm{~m}, 2 \mathrm{H}), 1.05-$ $0.91(\mathrm{~m}, 2 \mathrm{H})$.

${ }^{13} \mathrm{C}$ NMR (100 MHz, $\left.\mathrm{CDCl}_{3}\right): \delta 138.7,128.3,127.7,127.4,82.3,77.2,73.9,73.3,43.1$, $29.3,28.7,28.6,28.2,26.7,26.3,26.2,23.5$.

FTIR (neat) $v_{\max }: 3113,3088,3063,3030,2926,2853,1497,1450,1113,1086,737,698$ $\mathrm{cm}^{-1}$.

HRMS (EI) $m / z$. Calcd for $\mathrm{C}_{19} \mathrm{H}_{28} \mathrm{O}_{2}\left[\mathrm{M}^{+}\right]=288.2084$. Found: 288.2082 . 


\section{4-(4-Chloro-6-cyclohexyl-tetrahydro-2H-pyran-2-yl)butan-1-ol (3a)}

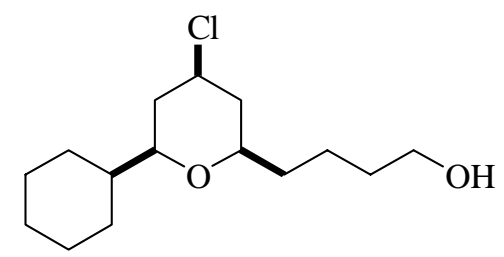

Colourless oil (46 mg, 60\% yield).

${ }^{1} \mathrm{H}$ NMR (400 MHz, $\left.\mathrm{CDCl}_{3}\right): \delta 3.99(\mathrm{tt}, J=11.7,4.5 \mathrm{~Hz}, 1 \mathrm{H}), 3.65$ (t, $\left.J=6.3 \mathrm{~Hz}, 2 \mathrm{H}\right)$, 3.28-3.19 (m, 1H), 2.97 (ddd, $J=11.2,7.1,1.3 \mathrm{~Hz}, 1 \mathrm{H}), 2.15(\mathrm{tdd}, J=12.3,4.5,2.1 \mathrm{~Hz}$, $1 \mathrm{H}), 2.10(\mathrm{tdd}, J=2.2,4.6,12.7 \mathrm{~Hz}, 1 \mathrm{H}), 1.97-1.87(\mathrm{~m}, 1 \mathrm{H}), 1.78-1.69(\mathrm{~m}, 2 \mathrm{H}), 1.68-$ $1.54(\mathrm{~m}, 6 \mathrm{H}), 1.51-1.35(\mathrm{~m}, 7 \mathrm{H}), 1.27-1.14(\mathrm{~m}, 2 \mathrm{H}), 1.01-0.92(\mathrm{~m}, 2 \mathrm{H})$.

${ }^{13} \mathrm{C}$ NMR (100 MHz, $\left.\mathrm{CDCl}_{3}\right): \delta 81.1,76.5,62.8,56.8,42.8,42.7,39.7,35.5,32.5,29.2$, 28.7, 26.5, 26.1, 26.0, 21.8.

FTIR (neat) $v_{\max }: 3418,2930,2853,1450,1265,1086,1065,738,704,608 \mathrm{~cm}^{-1}$.

HRMS (EI) $m / z$ Calcd for $\mathrm{C}_{15} \mathrm{H}_{27} \mathrm{ClO}_{2}\left[\mathrm{M}^{+}\right]=274.1694$. Found: 274.1698 .

\section{2-(4-(Benzyloxy)butyl)-6-cyclohexyl-tetrahydro-2H-pyran (3c)}

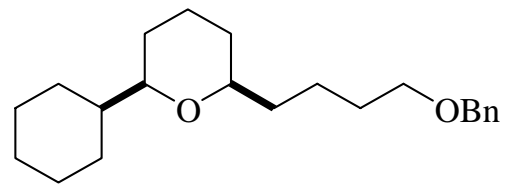

Colourless oil (61 mg, 66\% yield).

${ }^{1} \mathrm{H}$ NMR (400 MHz, $\left.\mathrm{CDCl}_{3}\right): \delta$ 7.37-7.31 (m, 4H), 7.30-7.23 (m, 1H), $4.50(\mathrm{~s}, 2 \mathrm{H}), 3.47$ $(\mathrm{t}, J=6.7 \mathrm{~Hz}, 2 \mathrm{H}), 3.24-3.14(\mathrm{~m}, 1 \mathrm{H}), 2.92(\mathrm{ddd}, J=11.2,7.8,1.6 \mathrm{~Hz}, 1 \mathrm{H}), 2.04-1.96$ (m, 1H), 1.86-1.78 (m, 1H), 1.75-1.67 (m, 2H), 1.55-1.49 (m, 3H), 1.41-1.37 (m, 4H), 1.33-1.22 (m, 7H), 1.18-1.07 (m, 3H), 0.92-0.82 (m, 2H).

${ }^{13} \mathrm{C}$ NMR $\left(100 \mathrm{MHz}, \mathrm{CDCl}_{3}\right): \delta 138.7,128.3,127.6,127.4,82.4,77.7,72.8,70.5,43.2$, 36.4, 32.0, 29.7, 29.4, 28.9, 28.6, 26.7, 26.2, 26.1, 23.9, 22.3.

FTIR (neat) $v_{\max }: 3109,3088,3063,3030,2910,2851,1495,1092,1045,733,696 \mathrm{~cm}^{-1}$. HRMS (EI) $m / z$ Calcd for $\mathrm{C}_{22} \mathrm{H}_{34} \mathrm{O}_{2}\left[\mathrm{M}^{+}\right]=330.2553$. Found: 330.2547 . 


\section{2,6-Dicyclohexyl-tetrahydro-2H-pyran (4)}

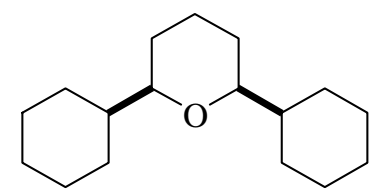

Colourless oil (55 mg, 79\% yield).

${ }^{1} \mathrm{H}$ NMR (400 MHz, $\left.\mathrm{CDCl}_{3}\right) \delta 2.89(\mathrm{ddd}, J=11.0,7.9,1.4 \mathrm{~Hz}, 2 \mathrm{H}), 2.07-1.96(\mathrm{~m}, 2 \mathrm{H})$, $1.88-1.80(\mathrm{~m}, 1 \mathrm{H}), 1.74-1.71(\mathrm{~m}, 2 \mathrm{H}), 1.71-1.66(\mathrm{~m}, 3 \mathrm{H}), 1.66-1.60(\mathrm{~m}, 4 \mathrm{H}), 1.30-1.22$ (m, 6H), 1.22-1.12 (m, 6H), 1.01-0.84 (m, 4H).

${ }^{13} \mathrm{C} \mathrm{NMR}\left(100 \mathrm{MHz}, \mathrm{CDCl}_{3}\right): \delta 82.4,43.3,29.4,29.0,28.9,26.7,26.3,26.1,24.0$.

FTIR (neat) $v_{\max }: 2920,2849,1449,1263,1084,1072,1045,743 \mathrm{~cm}^{-1}$.

HRMS (EI) $m / z$ Calcd for $\mathrm{C}_{17} \mathrm{H}_{30} \mathrm{O}\left[\mathrm{M}^{+}\right]$: 250.2297. Found: 250.2291.

\section{2-Cyclohexyl-6-phenyl-tetrahydro-2H-pyran (5)}

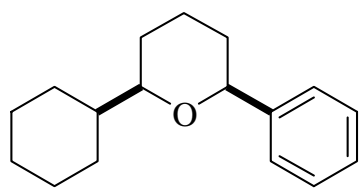

Colourless oil (45 mg, 66\% yield).

${ }^{1} \mathrm{H}$ NMR (400 MHz, $\left.\mathrm{CDCl}_{3}\right): \delta 7.40-7.30(\mathrm{~m}, 4 \mathrm{H}), 7.26-7.21(\mathrm{~m}, 1 \mathrm{H}), 4.35(\mathrm{dd}, J=11.1$, $1.8 \mathrm{~Hz}, 1 \mathrm{H}), 3.24$ (ddd, $J=11.1,6.2,1.7 \mathrm{~Hz}, 1 \mathrm{H}), 2.01-1.93(\mathrm{~m}, 2 \mathrm{H}), 1.90-1.82$ (m, 1H), 1.79-1.72 (m, 3H), 1.71-1.62 (m, 3H), 1.52-1.42 (m, 2H), 1.35-1.17 (m, 4H), 1.15-1.02 (m, 2H).

${ }^{13} \mathrm{C}$ NMR $\left(100 \mathrm{MHz}, \mathrm{CDCl}_{3}\right): \delta 144.0,128.1,126.9,125.7,82.5,79.4,43.3,33.9,29.1$, 28.7, 27.8, 26.7, 26.4, 26.3, 24.2.

FTIR (neat) $v_{\max }: 3088,3061,3028,2927,2851,1497,1450,1092,1043,749,698 \mathrm{~cm}^{-1}$. HRMS (EI) $m / z$ Calcd for $\mathrm{C}_{17} \mathrm{H}_{24} \mathrm{O}\left[\mathrm{M}^{+}\right]=244.1822$. Found: 244.1830 . 


\section{Procedures and Characterization of Intermediates of (+)-SCH 351448}

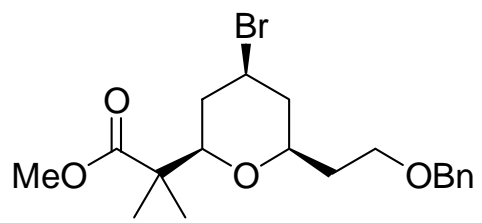

Methyl 2-((2R,4S,6R)-6-(2-(benzyloxy)ethyl)-4-bromo-tetrahydro-2H-pyran-2-yl)-2methylpropanoate (7): To a solution of homoallylic alcohol $6(0.88 \mathrm{~g}, 5 \mathrm{mmol})$ in dichloromethane $(50 \mathrm{~mL})$ was added $\operatorname{InBr}_{3}(0.18 \mathrm{~g}, 0.5 \mathrm{mmol}, 0.1$ equiv. $)$ at $0{ }^{\circ} \mathrm{C}$. The solution was allowed to cool to $-78{ }^{\circ} \mathrm{C}$ and trimethylsilyl bromide $(0.78 \mathrm{~mL}, 6 \mathrm{mmol}, 1.2$ equiv.) was added dropwise. The solution was stirred for 1 minute and was treated with a solution of 3-(benzyloxy)propanal in dichloromethane $(5 \mathrm{~mL})$. The reaction mixture was allowed to stir at $-78{ }^{\circ} \mathrm{C}$ for 4 hours, and was allowed to warm up gradually to room temperature. The reaction was allowed to proceed for another 12 hours prior to quenching with saturated sodium bicarbonate solution $(15 \mathrm{~mL})$. The aqueous layer was extracted with diethyl ether $(3 \times 30 \mathrm{~mL})$, and the combined organic extracts were washed with water $(50 \mathrm{~mL})$ and brine $(50 \mathrm{~mL})$ and dried over anhydrous magnesium sulphate, filtered and concentrated in vacuo. The residual crude product was purified by flash column chromatography (hexane / ether, 25:1) to afford the 4-bromo-THP product as colorless oil (1.30 g, 65\% yield, $91 \%$ ee ).

$\mathrm{R}_{\mathrm{f}}=0.59$ (4:1 hexane/ethyl acetate).

${ }^{1} \mathrm{H}$ NMR (500 MHz, $\left.\mathrm{CDCl}_{3}\right): \delta 7.33(\mathrm{~m}, 5 \mathrm{H}), 4.47,(\mathrm{~s}, 2 \mathrm{H}), 4.15$, (tt, $J=12.0,4.4 \mathrm{~Hz}$, $1 \mathrm{H}), 3.64(\mathrm{~s}, 3 \mathrm{H}), 3.55-3.51(\mathrm{~m}, 1 \mathrm{H}) 3.51(\mathrm{dd}, J=11.7,4.1 \mathrm{~Hz}, 1 \mathrm{H}), 3.50(\mathrm{t}, J=5.7 \mathrm{~Hz}$, 2H), $2.19(\mathrm{tdd}, J=12.4,4.4,2.1 \mathrm{~Hz}, 1 \mathrm{H}), 2.12(\mathrm{tdd}, J=12.4,4.4,2.1 \mathrm{~Hz}, 1 \mathrm{H}), 1.77-1.67$ (m, 4H), $1.17(\mathrm{~s}, 3 \mathrm{H}), 1.12(\mathrm{~s}, 3 \mathrm{H})$.

${ }^{13} \mathrm{C}$ NMR $\left(125 \mathrm{MHz}, \mathrm{CDCl}_{3}\right): \delta 176.6,138.4,128.4,127.7,127.6,81.5,74.6,73.1,66.5$, 51.9, 47.0, 46.5, 43.4, 37.6, 35.9, 20.8, 20.6.

FTIR (neat) $v_{\max }: 2949,1732,1275,1194,1070,737,698 \mathrm{~cm}^{-1}$.

HRMS (EI) $m / z$ Calcd for $\mathrm{C}_{19} \mathrm{H}_{27} \mathrm{BrO}_{4}\left[\mathrm{M}^{+}\right]=398.1087$. Found: 398.1077.

$[\alpha]^{23}=+4.2\left(c=1.65, \mathrm{CH}_{2} \mathrm{Cl}_{2}\right)$ 
The enantiomeric excess was determined by HPLC analysis employing Daicel Chiracel ODH column ( $n$-hexane : $i$-propanol 99:1, $1.0 \mathrm{~mL} / \mathrm{min}): \mathrm{t}_{1}=8.5 \mathrm{~min}($ minor $), \mathrm{t}_{2}=14.1$ $\min$ (major).

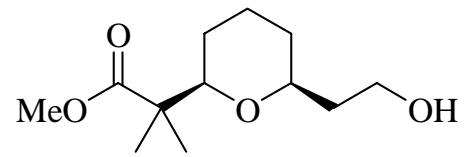

Methyl 2-((2R,6S)-tetrahydro-6-(2-hydroxyethyl)-2H-pyran-2-yl)-2-methylpropanoate (8): To an oven-dried $100 \mathrm{~mL}$ round-bottom flask equipped with a magnetic stirring bar was added $\mathrm{Pd} / \mathrm{C}(10 \% \mathrm{w} / \mathrm{w}, 2.22 \mathrm{~g}, 0.3$ equiv.) and was flushed with nitrogen before cooling to $0{ }^{\circ} \mathrm{C}$. Methanol $(63 \mathrm{~mL})$ was added slowly to the solid while stirring at a minimum speed of $500 \mathrm{rpm}$. The suspension was allowed to warm up to room temperature and was added an ethyl acetate $(7 \mathrm{~mL})$ solution of 4-bromo-THP methyl ester 7 (2.80 g, $7 \mathrm{mmol})$. The mixture was allowed to proceed under a $\mathrm{H}_{2}$ atmosphere introduced through a balloon for 8 hours. The reaction mixture was subsequently treated with $\mathrm{NaHCO}_{3}$ (1.76 g, $21 \mathrm{mmol}, 3$ equiv.) and was allowed to stir under $\mathrm{H}_{2}$ atmosphere for another 16 hours. The mixture was filtered through a pad of Celite and flushed with $300 \mathrm{~mL}$ of dichloromethane. The solution was concentrated in vacuo and was dissolved in $50 \mathrm{~mL}$ diethyl ether. The organic layer was washed with $\mathrm{H}_{2} \mathrm{O}(3 \times 20 \mathrm{~mL})$ and brine $(20 \mathrm{~mL})$ and dried over anhydrous magnesium sulphate, filtered and concentrated in vасио. The residual crude product was purified by flash column chromatography (hexane / ethyl acetate, 8:1) to afford the debromo-THP-alcohol as colorless oil (1.10 g, 68\% yield).

$\mathrm{R}_{\mathrm{f}}=0.13$ (4:1 hexane/ethyl acetate).

${ }^{1} \mathrm{H}$ NMR (500 MHz, $\left.\mathrm{CDCl}_{3}\right): \delta$ 3.73-3.69 (m, 2H), 3.68 (s, 3H), 3.58-3.52 (m, 2H), 2.80 (br s, 1H), 1.90-1.84 (m, 1H), 1.77-1.73 (m, 1H), 1.71-1.67 (m, 2H), 1.53-1.47 (m, 2H), 1.31-1.23 (m, 2H), $1.16(\mathrm{~s}, 3 \mathrm{H}), 1.12(\mathrm{~s}, 3 \mathrm{H})$.

${ }^{13} \mathrm{C}-\mathrm{NMR}(125 \mathrm{MHz}, \mathrm{CDCl} 3): \delta 177.5,83.0,79.3,61.6,51.9,46.6,38.0,31.5,24.8,23.4$, 21.9, 19.7 .

FTIR (neat) $v_{\max }: 3446,2943,2860,1732,1437,1271,1088,1047 \mathrm{~cm}^{-1}$.

HRMS (EI) $m / z$ Calcd for $\mathrm{C}_{12} \mathrm{H}_{22} \mathrm{O}_{4}\left[\mathrm{M}^{+}\right]=230.1513$. Found 230.1507. 
$[\alpha]^{23}{ }_{\mathrm{D}}=+8.8\left(c=1.55, \mathrm{CH}_{2} \mathrm{Cl}_{2}\right)$ 